\title{
Video Article \\ Isolation and Flow Cytometric Analysis of Immune Cells from the Ischemic Mouse Brain
}

\author{
Claudia Pösel ${ }^{1}$, Karoline Möller ${ }^{*}$, Johannes Boltze ${ }^{1,2}$, Daniel-Christoph Wagner ${ }^{1}$, Gesa Weise ${ }^{1,3}$ \\ ${ }^{1}$ Ischemia Research Unit, Fraunhofer Institute for Cell Therapy and Immunology \\ ${ }^{2}$ Fraunhofer Research Institution for Marine Biotechnology, University of Lubeck \\ ${ }^{3}$ Department of Neurology, University of Leipzig \\ * These authors contributed equally
}

Correspondence to: Gesa Weise at gesa.weise@izi.fraunhofer.de

URL: https://www.jove.com/video/53658

DOI: doi:10.3791/53658

Keywords: Immunology, Issue 108, Ischemic stroke, brain inflammation, repair, cell isolation, flow cytometry, quantification, characterization, cell phenotyping, immune cells, leukocytes

Date Published: 2/12/2016

Citation: Pösel, C., Möller, K., Boltze, J., Wagner, D.C., Weise, G. Isolation and Flow Cytometric Analysis of Immune Cells from the Ischemic Mouse Brain. J. Vis. Exp. (108), e53658, doi:10.3791/53658 (2016).

\section{Abstract}

Ischemic stroke initiates a robust inflammatory response that starts in the intravascular compartment and involves rapid activation of brain resident cells. A key mechanism of this inflammatory response is the migration of circulating immune cells to the ischemic brain facilitated by chemokine release and increased endothelial adhesion molecule expression. Brain-invading leukocytes are well-known contributing to earlystage secondary ischemic injury, but their significance for the termination of inflammation and later brain repair has only recently been noticed.

Here, a simple protocol for the efficient isolation of immune cells from the ischemic mouse brain is provided. After transcardial perfusion, brain hemispheres are dissected and mechanically dissociated. Enzymatic digestion with Liberase is followed by density gradient (such as Percoll) centrifugation to remove myelin and cell debris. One major advantage of this protocol is the single-layer density gradient procedure which does not require time-consuming preparation of gradients and can be reliably performed. The approach yields highly reproducible cell counts per brain hemisphere and allows for measuring several flow cytometry panels in one biological replicate. Phenotypic characterization and quantification of brain-invading leukocytes after experimental stroke may contribute to a better understanding of their multifaceted roles in ischemic injury and repair.

\section{Video Link}

The video component of this article can be found at https://www.jove.com/video/53658/

\section{Introduction}

Cerebral stroke triggers a sustained inflammatory response that starts rapidly after cessation of the local blood flow and involves virtually all parts of the immune system. One important hallmark of this inflammatory response is a timed influx of immune cells to the brain which is driven by the activation of brain endothelial cells, substantial chemokine secretion and increased sympathetic outflow ${ }^{1-3}$. Inflammatory cell infiltration was previously considered deleterious in ischemic stroke, however, several treatment trials designed to indiscriminately block leukocyte egress to the brain failed to induce a measurable clinical benefit ${ }^{4}$. More recently, it became evident that monocyte-derived cells initially involved in progression of ischemic damage ${ }^{5}$ might also play a pivotal role for resolution of inflammation and subsequent tissue repair ${ }^{6}$.

Thanks to the identification of phenotypic and functional heterogeneity among monocytes and macrophages, the knowledge on the role of mononuclear phagocytes in development and resolution of inflammation has significantly expanded. In mice, circulating monocytes can be classified into at least two functionally distinct subsets according to their surface expression of lymphocyte antigen 6 complex (Ly-6C) ${ }^{7}$. While Ly-6C ${ }^{\text {high }}$ 'inflammatory monocytes' were clearly shown to be essential for the control of bacterial infections ${ }^{8}$, their role in sterile injury is more controversial. In ischemic stroke, selective ablation of CCR2+ Ly-6C ${ }^{\text {high }}$ monocytes resulted in hemorrhagic infarct transformation ${ }^{6}$. However, the same experimental approach improved acute disability after intracerebral hemorrhage ${ }^{9}$. Similarly, different subsets of T cells are believed to exert either detrimental ${ }^{10}$ or protective actions ${ }^{11}$ in ischemic brain injury, however, data is controversial ${ }^{12,13}$ and warrants further investigations. Given this increasing complexity, it becomes clear that deeper knowledge on the roles of the diverse immune cells in ischemic injury and repair is crucial for translating experimental findings into therapies targeting post stroke inflammation.

Today, the most powerful tool for analyzing cellular immune responses is polychromatic flow cytometry. It allows the identification and quantification of various immune cell subsets at the site of inflammation without the need to bias the system by in vivo labeling or genetic manipulation ${ }^{14}$. Simultaneous staining of cell surface markers with antibodies against intracellular cytokines ${ }^{15}$ or transcription factors $^{16}$ additionally provides information about the functional state of individual, phenotypically identified cells. As one major drawback, single cell suspensions are required for flow cytometric assays and thus information on localization of cellular infiltrates is lost. However, while histology is 
ideal to gain spatial information, it is limited by the number of antibodies that can be used at a single time to characterize immune cell subtypes in a particular tissue. Today, a combination of the presence and absence of various surface markers is needed to unambiguously identify rare immune cell subsets, especially the distinct monocyte-derived cell populations during inflammation ${ }^{17}$.

Here, we describe an efficient protocol to isolate high numbers of leukocytes from the postischemic mouse brain using a simple single-layer density gradient. The obtained cell suspensions can be either analyzed by multi-dimensional flow cytometry or further be enriched by flow cytometric sorting or immunomagnetic selection to perform highly specific downstream analyses. The method details transcardial perfusion, removal of brain hemispheres, dissociation of cerebral tissue into single cell suspensions, density gradient centrifugation for myelin removal as well as antibody staining for flow cytometric analysis.

\section{Protocol}

All animal experiments must conform to according standards for animal care (e.g., the Guide for the Care and Use of Laboratory Animals published by the US National Institutes of Health, NIH Publication No. 85-23, revised 1996) and have to be approved by the appropriate state authority.

\section{Preparation of Reagents}

1. Digestion buffer (1 $\mathrm{ml}$ per hemisphere): Dissolve a standardized mixture of purified digestive enzymes, e.g., Liberase with low thermolysin concentration (TL) to a concentration of $2 \mathrm{U} / \mathrm{ml}$ in Hanks Balanced Salt Solution (HBSS) containing calcium (Ca) and magnesium (Mg)

2. Washing buffer with DNAse: Dissolve DNAse I to a concentration of $666 \mathrm{U} / \mathrm{ml}$ in HBSS (Ca/Mg free) containing $10 \%$ of fetal calf serum (FCS)

3. Washing buffer without DNAse: HBSS (Ca/Mg free) containing $10 \%$ of FCS

4. Density gradient medium ( $5 \mathrm{ml}$ per hemisphere): prepare stock isotonic density gradient medium (SIP; $100 \%)$ by mixing nine parts of density gradient medium with one part $1.5 \mathrm{M}$ sodium chloride. Dilute SIP to $25 \%$ density by adding an appropriate volume of $\mathrm{HBSS}$ (Ca/Mg free) containing $3 \%$ of FCS

5. Flow cytometry (FC) buffer: Prepare phosphate buffered saline (PBS) containing $3 \%$ of FCS

\section{Transient Middle Cerebral Artery Occlusion}

NOTE: Transient middle cerebral artery occlusion (MCAO) via the intraluminal suture technique was performed as described previously ${ }^{18}$ in $12-$ weeks old male C57BL/6 mice.

1. Briefly, anesthetize mice with $2.0 \%$ isoflurane in $100 \%$ oxygen. Maintain the body temperature at $36.5^{\circ} \mathrm{C} \pm 0.5^{\circ} \mathrm{C}$ by a feedback-controlled heating device.

2. After ligation of the common carotid artery and the external carotid artery, introduce a standardized silicon-rubber coated monofilament in the common carotid artery and advance it to the origin of the middle cerebral artery.

3. After $45 \mathrm{~min}$ remove the filament to allow reperfusion. In sham-operated animals, immediately withdraw the filament after occluding the middle cerebral artery to avoid ischemia.

\section{Transcardial Perfusion and Brain Dissection}

1. Prepare a peristaltic perfusion pump by immersing one end of the tubing into ice-cold HBSS (Ca/Mg free). Fix a blunt $23 \mathrm{G}$ needle to the other end of the perfusion tube and switch on the pump to completely fill the tubing with $\mathrm{HBSS}(\mathrm{Ca} / \mathrm{Mg}$ free).

2. Deeply anesthetize mouse with $4 \%$ isoflurane and euthanize by $\mathrm{CO}_{2}$ inhalation.

3. Place mouse dorsally on a dissection board embedded in a plastic tray. Spread fore and hind paws as wide as possible and fix them on the dissection board with $20 \mathrm{G}$ needles.

4. Grab abdominal skin with a straight $1 \times 2$ teeth forceps and use sharp iris scissors to make a lateral incision through the integument and abdominal wall and expose the liver.

5. Lift the sternum and incise the diaphragm with the blunt blade of sharp/blunt iris scissors. Continue to cut the lateral rib cage at both sides in caudocranial direction. Be careful not to injure the lung, the heart and the thoracic arteries.

6. Lift the skin flap with a blunt forceps and pin it on the dissection board. Use blunt-end forceps and scissors to carefully separate the heart from connective tissue.

7. Hold heart with a blunt-end forceps and insert the tip of the blunt $23 \mathrm{G}$ needle with the attached perfusion tube into the apex of the left ventricle. Note: Do not insert the needle too far into the left ventricle to avoid injury of the interventricular septum. If necessary, fix the cannula in place with a straight sharp forceps.

8. Incise the right atrium with sharp iris scissors and immediately turn on the pump (flow rate: $8-10 \mathrm{ml} / \mathrm{min}$ ).

9. Continue perfusion until the liver shows a light coffee color ( $\sim 30 \mathrm{ml}$ of HBSS). Throughout the perfusion, carefully avoid any air bubble formation in the tubing.

10. Decapitate the mouse with straight surgical scissors just behind the skull. Use iris scissors to make a midline incision of the scalp to expose the skull.

11. Place one tip of sharp iris scissors into the foramen magnum and cut laterally into the skull. Repeat for the other side.

12. Use sharp iris scissors to carefully cut from the same cavity up the midline towards the nose. Try to keep the end of the scissors as superficial as possible to avoid injury of the brain.

13. Use fine forceps to gently peel the cranial bones from each brain hemisphere. Note: Infarcted tissue may adhere to the meninges or the skull; make sure not to lose brain tissue due to incautious dissection

14. Lift the brain with a spatula and use sharp iris scissors to carefully dissect cranial nerve fibers that fix it to the skull. Place the brain into a 15 $\mathrm{ml}$ tube filled with $10 \mathrm{ml}$ of HBSS $(+\mathrm{Ca} / \mathrm{Mg})$ and keep it shortly on ice. 


\section{Dissociation of Cerebral Tissue into Single Cell Suspensions}

1. Carefully remove brain stem and cerebellum with a clean razor blade. Use a clean razor blade to hemisect the brain and cut each hemisphere along the coronal plane into three pieces of roughly equal size.

2. Mince dissected tissue of each hemisphere through a $100 \mu \mathrm{m}$ cell strainer using the plunger end of a $5 \mathrm{ml}$ syringe. Continuously rinse the cell strainer with ice-cold HBSS $(+\mathrm{Ca} / \mathrm{Mg})$. Store homogenized samples on ice.

3. Centrifuge at $286 \mathrm{xg}$ and $4^{\circ} \mathrm{C}$ for $5 \mathrm{~min}$, carefully discard the supernatant. Resuspend the pellet in $1 \mathrm{ml}$ of digestion buffer and transfer the suspension into a $2 \mathrm{ml}$ tube. Incubate suspension under slow continuous rotation at $37^{\circ} \mathrm{C}$ for $1 \mathrm{hr}$.

4. Sieve cell suspension through a $70 \mu \mathrm{m}$ cell strainer and rinse thoroughly with $3 \mathrm{ml}$ of washing buffer containing DNAse followed by $15 \mathrm{ml}$ of DNAse-free washing buffer. Centrifuge at $286 \mathrm{xg}$ and $18^{\circ} \mathrm{C}$ for $5 \mathrm{~min}$ and discard the supernatant. Note: Note: The use of DNAse eliminates DNA mucus caused by cell lysis which might lead to cell aggregation compromising cell survival.

\section{Density Gradient Centrifugation for Removal of Myelin and Cell Debris}

1. Resuspend cell pellet in $5 \mathrm{ml}$ of $25 \%$ density gradient medium and pipette the suspension to a $15 \mathrm{ml}$ tube. Mix thoroughly by repeated and gentle pipetting avoiding bubble formation. Note: density gradient medium should be used at RT to prevent cell clumping.

2. Centrifuge at $521 \mathrm{xg}$ and $18{ }^{\circ} \mathrm{C}$ for $20 \mathrm{~min}$. Use lowest acceleration profile of the rotor and allow the rotor to stop without brake. Gently remove the tubes from the centrifuge without shaking. Carefully aspirate the myelin coat and the supernatant while preserving the cell pellet at the bottom of tube. Note: Make sure to remove the entire myelin coat as any leftover will impair flow cytometric analysis.

3. Resuspend the pellet in $10 \mathrm{ml}$ of DNAse free washing buffer and pipette the suspension to a new $15 \mathrm{ml}$ tube. Note:This washing step is crucial since sufficient removal of density gradient medium significantly contributes to the quantity and quality of the final cell sample.

4. Centrifuge again at $286 \mathrm{xg}$ and $10^{\circ} \mathrm{C}$ for $5 \mathrm{~min}$ and discard the supernatant. Resuspend cells in $100 \mu \mathrm{l}$ of cold washing buffer and determine cell counts and viability by trypan blue exclusion in a hemocytometer. Store samples at $4{ }^{\circ} \mathrm{C}$ and further process them quickly.

\section{Antibody Staining for Flow Cytometric Analysis}

1. Prior to antibody labeling, incubate the cell suspension at $4{ }^{\circ} \mathrm{C}$ for 10 min with anti-murine CD16/CD32 FC-Receptor blocking reagent (final concentration of $2.5 \mu \mathrm{g} / \mathrm{ml}$, dilution factor $1: 200$ ) to prevent unspecific binding.

2. Add fluorophore-conjugated primary antibodies at the appropriate concentration (as indicated in Table 1) to the cell suspension and incubate at $4{ }^{\circ} \mathrm{C}$ for $20 \mathrm{~min}$ in the dark. Note: Control samples are not stained with primary antibodies, but otherwise treated the same.

3. Wash cells with $2 \mathrm{ml}$ of $\mathrm{FC}$ buffer and centrifuge at $350 \times \mathrm{g}$ and $10^{\circ} \mathrm{C}$ for $7 \mathrm{~min}$.Carefully aspirate the supernatant and resuspend cell pellet in $200 \mu \mathrm{l}$ of $\mathrm{FC}$ buffer. Store samples temporarily at $4{ }^{\circ} \mathrm{C}$ in the dark until flow cytometric analysis.

\section{Absolute Quantification by Counting Beads}

1. Reversely pipette $40 \mu \mathrm{l}$ of FC buffer and $10 \mu \mathrm{l}$ of the cell suspension into a counting tube containing a known number of fluorescent beads Note: Pay attention that pipettes are calibrated to deliver exactly $10 \mu \mathrm{l}$ of sample as subsequent calculation of leukocyte counts refers to this volume.

2. Incubate cell suspension with FITC-labeled CD45 antibody (final concentration of $5 \mu \mathrm{g} / \mathrm{ml}$, dilution factor $1: 100$ ) at $4{ }^{\circ} \mathrm{C}$ for 20 min in the dark.

3. Reversely fill the counting tube with $200 \mu \mathrm{l}$ of FC buffer without any washing step and directly record CD45+ cells in the flow cytometer.

\section{Flow Cytometric Acquisition}

Note: To analyze the proposed antibody panel, the appropriate cytometer has to be equipped with a blue (488 $\mathrm{nm})$, red $(635 \mathrm{~nm})$ and violet (405 $\mathrm{nm}$ ) laser and filters for FITC, PE, PerCP Cy5.5, PECy7, APC-Cy7 and AmCyan.

1. Adjust forward (FSC) and sideward (SSC) scatter by using unstained cells from an ischemic hemisphere. Discriminate doublets from single cells in a dot plot showing the area and height of FSC.

2. Display all single cells in single-parameter histograms for each fluorescence channel used and adjust the photomultiplier tube (PMT) voltages so that single cells are displayed in the far left of the histogram.

3. Use a CD45-FITC single stained sample to check scatter and PMT settings for the cells of interest by backgating CD45 ${ }^{\text {high }}$ immune cells in each histogram and scatter plot. Note: Adapt PMT voltages of FITC so that CD45 negative, intermediate (int) and high expressing cells can be differentiated from each other.

4. Use antibody captured compensation beads to perform multi-color compensation. Set gates for microglia $\left(C D 45^{\text {int }}\right)$ and leukocytes $\left(C D 45^{\text {high }}\right)$ and subsequently define leukocyte subpopulations according to the gating strategy displayed in Figure 1.

\section{Representative Results}

Cerebral ischemia was initiated in 12-weeks old male C57BL/6 mice via transient filament occlusion of the left middle cerebral artery (MCAO). For sham operation the filament was inserted to occlude the left middle cerebral artery and withdrawn immediately to allow instant reperfusion. Importantly, cellular neuroinflammation differs substantially among commonly applied stroke models ${ }^{19}$. This has to be kept in mind especially when extrapolating findings from animal studies to the heterogeneous pathophysiology of human stroke. 
Mice were sacrificed $24 \mathrm{hr}$ after MCAO to analyze early immune cell invasion to the ischemic brain. Cell counts obtained from the ischemic hemisphere (MCAO ipsi; $0.95 \pm 0.25 \times 10 \mathrm{E} 6$ ) after density gradient centrifugation were comparable to those from the contralateral hemisphere (MCAO contra; $1.09 \pm 0.30 \times 10 \mathrm{E} 6$ ) and the ipsilateral hemisphere of sham-operated mice (sham ipsi; $1.12 \pm 0.18 \times 10 \mathrm{E} 6$, one-way ANOVA, $p=$ 0.524 ). Viability of the isolated cells measured by trypan blue exclusion was high and did not significantly differ among the groups (sham $96.85 \pm$ $0.60 \%$, MCAO contra $97.12 \pm 1.18 \%$, MCAO ipsi $95.68 \pm 2.04 \%$, one-way-ANOVA, $p=0.253$ ).

The composition of the brain infiltrate $24 \mathrm{hr}$ after MCAO was determined by flow cytometric analysis. Figure 1 illustrates the gating strategy schematically $(A)$ and in a representative stroke animal $(B)$. Brain-infiltrating leukocytes were defined as CD45 ${ }^{\text {high }}$ cells that can be differentiated from $C D 45^{\text {int }}$ microglia. Within the CD45 ${ }^{\text {high }}$ population, polymorphonuclear neutrophils (PMN) were identified by Ly-6G expression, while T lymphocytes were delineated as CD45 ${ }^{\text {high }}$ CD3+ cells. The remaining CD45 ${ }^{\text {high }}$ cells were then distinguished by CD19 (B lymphocytes) and CD11b expression. The CD11b+ fraction was further subcategorized into Ly- $6 C^{\text {high }}$ 'inflammatory monocytes' and a Ly- $6 C^{\text {low }}$ population that encompasses monocytes, dendritic cells (DC) and macrophages.

In the acute stage of stroke, myeloid immune cells dominate the brain infiltrate ${ }^{3,20}$. Neutrophils enter the brain rapidly after vessel occlusion and promote the extravasation of inflammatory monocytes ${ }^{21}$. Figure $2 \mathrm{~A}$ displays the percentage increase of Ly6-G+ neutrophils in the ischemic hemisphere $24 \mathrm{hr}$ after MCAO compared to the contralateral hemisphere and sham surgery. By contrast, the proportion of CD3+ T cells in the ischemic hemisphere is (relatively) lower than in the healthy brain. Within the CD11b+ population, brain ischemia shifts the balance towards a strong preponderance of Ly-6C ${ }^{\text {high }}$ inflammatory monocytes (Figure 2B).

In addition to relative distributions, counting tubes were used to determine absolute numbers of immune cell subsets in samples on the basis of CD45 positive events (Figure 3). Counting tubes contain a lyophilized pellet which releases a known number of fluorescent beads. The absolute number of positive cells in the sample can be determined by relating cellular events to bead events. To calculate the number of CD45 ${ }^{\text {high }}$ leukocytes per hemisphere the following equation was used: CD $45^{\text {high }}$ cells per hemisphere $=\left(C D 45^{\text {high }}\right.$ events $\mathrm{x}$ total counting beads/ sample bead events) $x$ (total suspension volume $(100 \mu \mathrm{l}) /$ sample volume $(10 \mu \mathrm{l})) .24 \mathrm{hr}$ after MCAO, total leukocyte counts in the infarct hemisphere were significantly increased as compared to the contralateral hemisphere and sham surgery (Figure 3D, one-way ANOVA, $p=0.0004)$. Counts of the distinct immune cell subsets (PMN, T-cells, B-cells, Ly- $6 C^{\text {high }}$ and Ly- $6 C^{\text {low }}$ monocytes) can be easily computed by multiplying their relative frequency with the total CD45 ${ }^{\text {high }}$ leukocyte number of the respective sample.

A

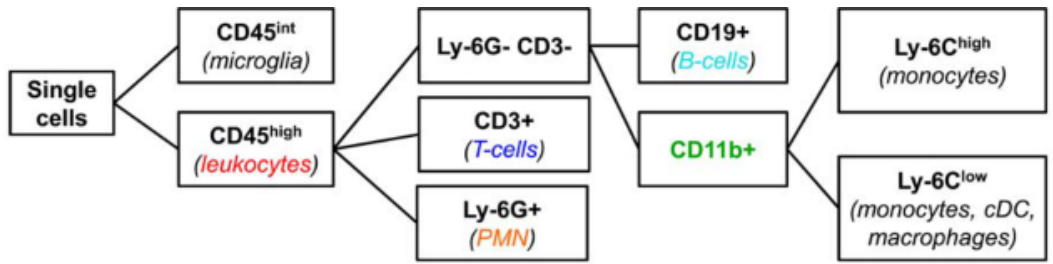

B
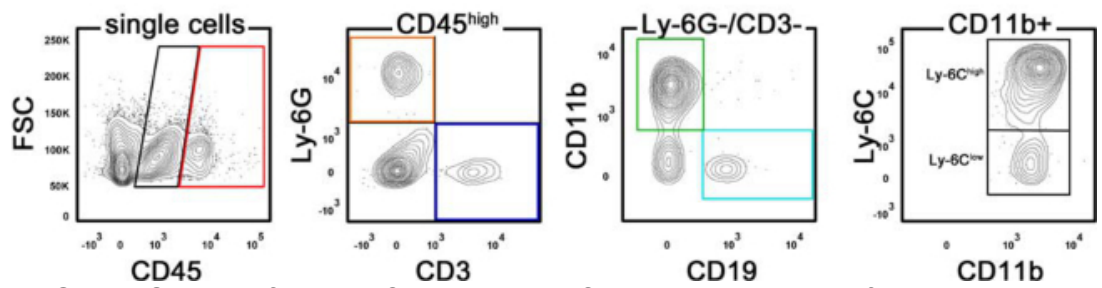

Figure 1. Gating Strategy for Flow Cytometry. (A) Schematic illustration of the gating strategy. (B) shows flow cytometric analysis of leukocytes isolated from a representative mouse brain $24 \mathrm{hr}$ after middle cerebral artery occlusion. FSC forward scatter, PMN polymorphonuclear neutrophils, CDC classical dendritic cells. Please click here to view a larger version of this figure. 


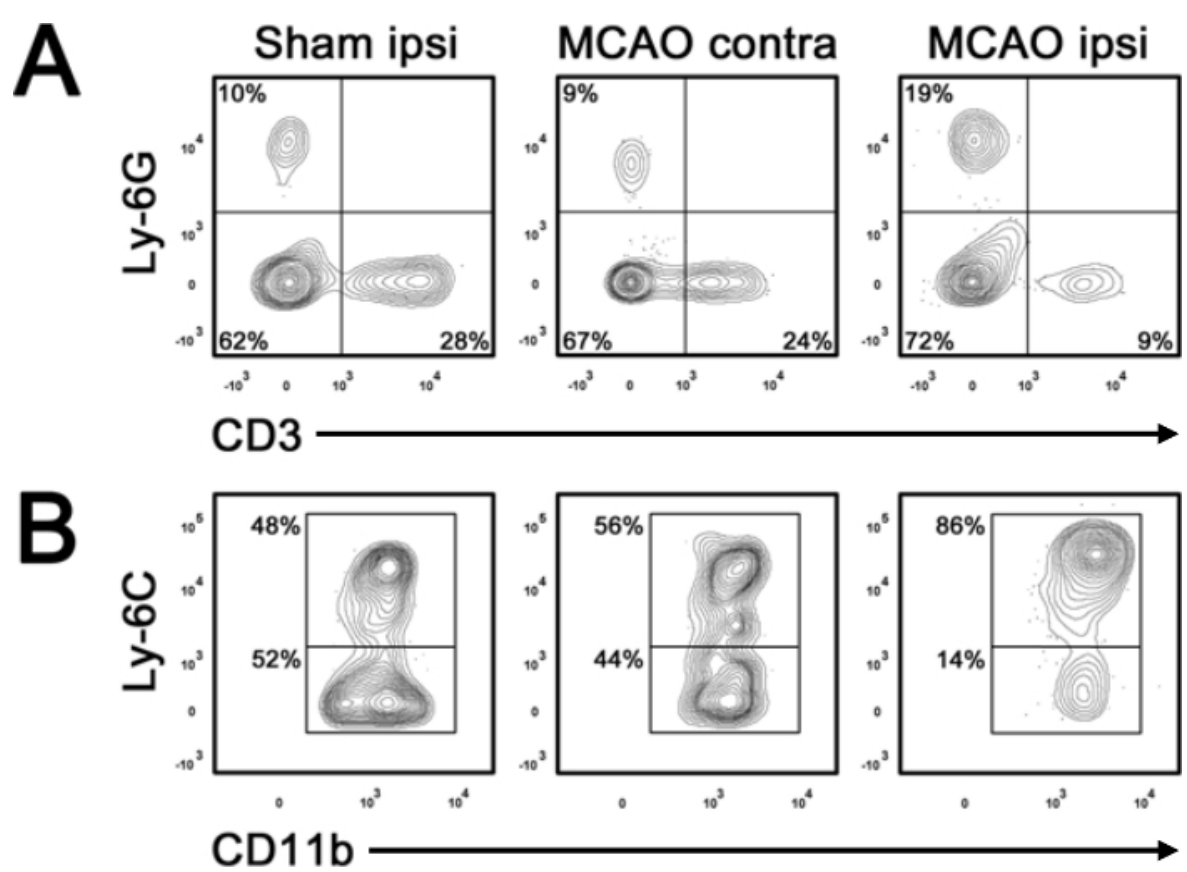

Figure 2. Differentiation of Immune Cell Subsets. Relative distribution of Ly-6G+-neutrophils (A), CD3+ T cells (A) and monocyte subsets identified by differential expression of Ly-6C (B) in the ipsilateral (ipsi) and contralateral (contra) hemisphere $24 \mathrm{hr}$ after middle cerebral artery occlusion (MCAO) or a respective sham surgery. Percentage of each population is indicated in the gate. Please click here to view a larger version of this figure. 

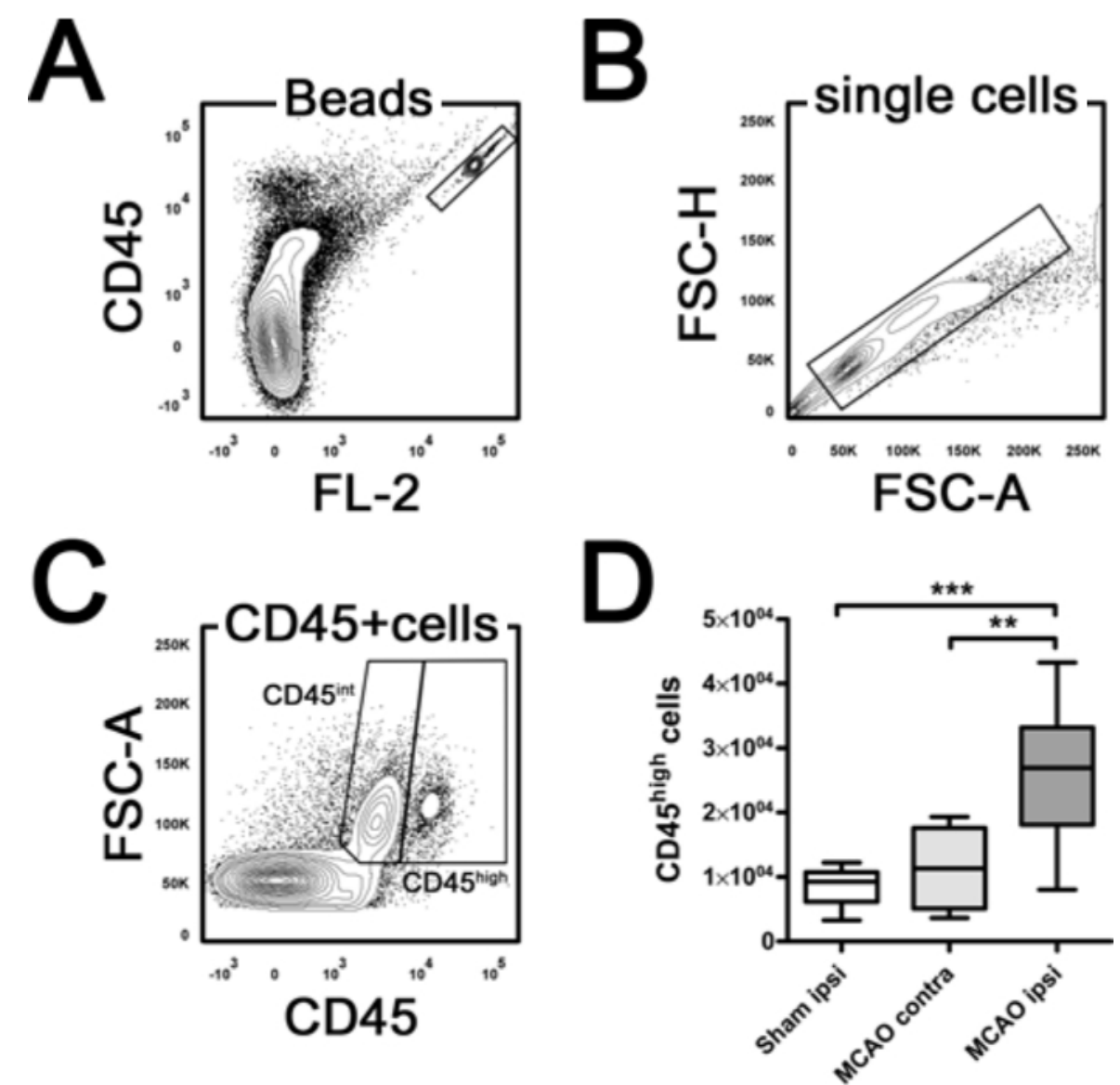

Figure 3. Quantification of Brain Leukocytes by Counting Beads. (A) shows the highly double-positive bead gate. Within the single cell population (B) CD45 ${ }^{\text {int }}$ microglia can be differentiated from CD45 $5^{\text {high }}$ leukocytes (C). For quantification, the number of gated CD45 ${ }^{\text {high }}$ events was normalized to the counted bead events. Note that total leukocyte $\left(C D 45^{\text {high }}\right)$ counts are significantly increased in the ipsilateral (ipsi) hemisphere compared to the contralateral (contra) hemisphere and sham surgery $24 \mathrm{hr}$ after middle cerebral artery occlusion (MCAO). ${ }^{* *} p<0.01,{ }^{* * *} p<0.001$ by one-way ANOVA and Tukey`s post hoc multiple comparisons test. $n=4-6$ per group. FSC forward scatter. Please click here to view a larger version of this figure.

\begin{tabular}{|c|c|c|c|c|c|c|}
\hline \multirow[t]{2}{*}{ Fluorochrome } & \multirow[t]{2}{*}{ FITC } & \multirow[t]{2}{*}{ PE } & PerCP & \multirow[t]{2}{*}{ PC7 } & \multirow[t]{2}{*}{ APC-Cy7 } & \multirow[t]{2}{*}{ V500 } \\
\hline & & & (Cy5.5) & & & \\
\hline Antigen & CD45.2 & CD3 & Ly-6G & CD19 & Ly-6C & CD11b \\
\hline $\begin{array}{l}\text { Final } \\
\text { concentration }[\mu \mathrm{g} / \\
\mathrm{ml}]\end{array}$ & 5 & 1 & 0.2 & 0.2 & 1 & 1 \\
\hline Dilution factor & $1 / 100$ & $1 / 200$ & $1 / 1,000$ & $1 / 1,000$ & $1 / 200$ & $1 / 200$ \\
\hline Clone & 104 & $145-2 \mathrm{C} 11$ & $1 \mathrm{~A} 8$ & $6 \mathrm{D} 5$ & AL-21 & $\mathrm{M} 1 / 70$ \\
\hline
\end{tabular}

Table 1. Basic Antibody Cocktail for Immune Cell Identification in the Ischemic Brain.

\section{Discussion}

Here, we describe a simple and effective method for the isolation of leukocytes from the murine brain after experimental stroke. The approach reliably yields highly reproducible cell counts per brain hemisphere allowing to measure different flow panels in one biological replicate.

Apparently, an incomplete removal of blood including immune cells will result in a distorted view on the actual amount of inflammatory cells that entered the ischemic brain. Thus, when using this protocol pay particular attention to thorough transcardial perfusion to prevent contamination of infiltrated immune cells with non-inflammatory circulating leukocytes. From experience, the usage of blunt needles for left ventricle puncture reduces the risk for injury of the interventricular septum which would bypass perfusion of the systemic circulation. Emergence of perfusion fluid from the nostrils is a sign that the perfusion pressureis too high, thus, avoid flow rates $>10 \mathrm{ml} / \mathrm{min}$. Pale to white color of the brain tissue indicates good perfusion while pinkish color is a sign of poor perfusion. 
Another critical step of this protocol is efficient dissociation of brain tissue that comprises mechanical fragmentation as well as enzymatic digestion. Mincing the tissue through the cell strainer is critical to provide improved effectiveness of proteases. However, when homogenizing the tissue, avoid excessive pressure as mononuclear phagocytes are highly susceptible to autolysis ${ }^{22}$. For enzymatic digestion Liberase TL is recommended which is a mixture of highly purified collagenase I and II that contains low concentrations of the neutral protease thermolysin. The direct comparison with previously described isolation protocols ${ }^{14,22,23}$ revealed significantly higher recovery of viable cells by Liberase TL treatment (K.M. unpublished data). Compared to collagenase which is frequently used for the isolation of immune cells from the brain, Liberase TL contains negligible levels of endotoxin. This is of special importance if cells are sorted for downstream analysis because high levels of endotoxin might change the activation state of immune cells ${ }^{24}$ and severely impair cell culture outcomes ${ }^{25}$. Another drawback of traditional collagenase is significant lot-to-lot differences in the enzyme activities which jeopardizes reproducibility of results and requires determination of the working concentration for each $\operatorname{lot}^{26}$.

In the adult brain, myelin removal by density gradient centrifugation is an indispensable step for downstream applications such as flow cytometry or further studies on gene or protein expression. One major advantage of the protocol is the single-layer density procedure which does not require time-consuming preparation of gradients. Moreover, the separation protocol produces reliable results even when performed by rather inexperienced experimenters. It is devoid of layered gradients with densities close to one another that are difficult to pipette without disturbing the interface in between.

Based on the expression of the surface marker CD45, the protocol yields three major cell categories in the ischemic brain. The vast majority of cells belong to a CD45 negative population that is composed of neuronal cells, astroglia, ependymal and endothelial cells. Their abundant presence is attributed to the single-layer density gradient which primarily aims at efficient myelin and debris removal. Additionally, a CD45 ${ }^{\text {int }}$ population representing resident microglia ${ }^{27}$ can be differentiated from a CD45 ${ }^{\text {high }}$ population that primarily contains infiltrating hematogenous leukocytes. However, it should be noted that activated microglia may adopt the phenotype and function of blood-born myeloid cells. Thus, only

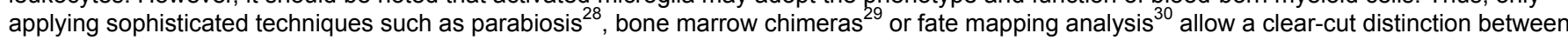
those two populations during inflammation.

Data analysis in flow cytometry is often limited to the percentage distribution of specific cell populations. However, when comparing the infarct hemisphere to non-infarcted brain tissue this information can be misleading because it does not consider total brain leukocyte counts which are changed by ischemic injury. Thus, to draw a full picture on the magnitude and phenotype of inflammatory infiltrates after stroke relative distribution of immune cell subsets should be complemented by absolute cell numbers. When using microbeads as described in this protocol, pipetting of a precise sample volume is absolutely mandatory to obtain reliable results. Moreover, reverse pipetting is strongly recommended to avoid foam formation in the counting tube. Arising air bubbles will reduce the expected volume of microbeads and hence lead to an overestimation of absolute CD45 ${ }^{\text {high }}$ leukocyte counts in the sample.

In summary, this protocol provides an easy and reliable approach to isolate immune cells from the brain. It may serve as a valuable tool to dissect the complexity of the inflammatory response to ischemic stroke. Future applications include investigations on the time-dependent role of monocyte subsets in propagation and resolution of ischemic brain inflammation. Similarly, the role of the adaptive immune system after stroke is poorly understood. Flow cytometric analysis of T cell subpopulations identified by the expression of subset-specific transcription factors or cytokines in situ might help to clarify their impact on long-term recovery after stroke and thus open promising avenues for the development of new treatment approaches.

\section{Disclosures}

The authors declare that they have no competing financial interests.

\section{Acknowledgements}

The authors thank Isabell Schulz for excellent technical support.

\section{References}

1. Courties, G., et al. Ischemic stroke activates hematopoietic bone marrow stem cells. Circulation research. 116 (3), $407-17$ (2015)

2. Iadecola, C., \& Anrather, J. The immunology of stroke: from mechanisms to translation. Nature medicine. 17 (7), $796-808$ (2011).

3. Möller, K.,Boltze, J., Pösel, C., Seeger, J., Stahl, T., \& Wagner, D.-C. Sterile inflammation after permanent distal MCA occlusion in hypertensive rats. Journal of cerebral blood flow and metabolism official journal of the International Society of Cerebral Blood Flow and Metabolism. 34 (2), 307-15 (2014).

4. Sughrue, M.E., Mehra, A., Connolly, E.S., \& D'Ambrosio, A.L. Anti-adhesion molecule strategies as potential neuroprotective agents in cerebral ischemia: a critical review of the literature. Inflammation research official journal of the European Histamine Research Society ... [et al.]. 53 (10), 497-508 (2004).

5. Dimitrijevic, O.B., Stamatovic, S.M., Keep, R.F., \& Andjelkovic, A.V. Absence of the chemokine receptor CCR2 protects against cerebral ischemia/reperfusion injury in mice. Stroke; a journal of cerebral circulation. 38 (4), 1345-53 (2007).

6. Gliem, M., et al. Macrophages prevent hemorrhagic infarct transformation in murine stroke models. Annals of neurology. 71 (6), $743-52$ (2012).

7. Geissmann, F., et al. Blood monocytes: distinct subsets, how they relate to dendritic cells, and their possible roles in the regulation of T-cell responses. Immunology and cell biology. 86 (5), 398-408 (2008).

8. Dunay, I.R., Fuchs, A., \& Sibley, L.D. Inflammatory monocytes but not neutrophils are necessary to control infection with Toxoplasma gondii in mice. Infection and immunity. 78 (4), 1564-70 (2010).

9. Hammond, M.D., et al. CCR2+ Ly6C(hi) inflammatory monocyte recruitment exacerbates acute disability following intracerebral hemorrhage. The Journal of neuroscience the official journal of the Society for Neuroscience. 34 (11), 3901-9 (2014). 
10. Shichita, T., et al. Pivotal role of cerebral interleukin-17-producing gammadeltaT cells in the delayed phase of ischemic brain injury. Nature medicine. 15 (8), 946-50 (2009).

11. Liesz, A., et al. Regulatory T cells are key cerebroprotective immunomodulators in acute experimental stroke. Nature medicine. 15 (2), $192-9$ (2009).

12. Kleinschnitz, C., \& Wiendl, H. Con: Regulatory T cells are protective in ischemic stroke. Stroke; a journal of cerebral circulation. 44 (8), e87-8 (2013).

13. Hu, X., Li, P., \& Chen, J. Pro: Regulatory T cells are protective in ischemic stroke. Stroke; a journal of cerebral circulation. 44 (8), e85-6 (2013).

14. Möller, K.,Stahl, T., Boltze, J., \& Wagner, D.-C. Isolation of inflammatory cells from rat brain tissue after stroke. Experimental \& translational stroke medicine. 4 (1), 20 (2012).

15. Foster, B., Prussin, C., Liu, F., Whitmire, J.K., \& Whitton, J.L. Detection of intracellular cytokines by flow cytometry. Current protocols in immunology / edited by John E. Coligan ... [et al.]. Chapter 6, Unit 6.24 (2007).

16. Albu, D.I., Califano, D., \& Avram, D. Flow cytometry analysis of transcription factors in T lymphocytes. Methods in molecular biology (Clifton, N.J.). 647, 377-90 (2010).

17. Prinz, M., Priller, J., Sisodia, S.S., \& Ransohoff, R.M. Heterogeneity of CNS myeloid cells and their roles in neurodegeneration. Nature neuroscience. 14 (10), 1227-35 (2011).

18. Wagner, D.-C., et al. Allometric dose retranslation unveiled substantial immunological side effects of granulocyte colony-stimulating factor after stroke. Stroke; a journal of cerebral circulation. 45 (2), 623-6 (2014).

19. Zhou, W., et al. Postischemic brain infiltration of leukocyte subpopulations differs among murine permanent and transient focal cerebral ischemia models. Brain pathology (Zurich, Switzerland). 23 (1), 34-44 (2013).

20. Gelderblom, M., et al. Temporal and spatial dynamics of cerebral immune cell accumulation in stroke. Stroke; a journal of cerebral circulation. 40 (5), 1849-57 (2009).

21. Soehnlein, O., \& Lindbom, L. Phagocyte partnership during the onset and resolution of inflammation. Nature reviews. Immunology. 10 (6), 427-39 (2010).

22. Cardona, A.E., Huang, D., Sasse, M.E., \& Ransohoff, R.M. Isolation of murine microglial cells for RNA analysis or flow cytometry. Nature protocols. 1 (4), 1947-51 (2006).

23. Arac, A., et al. Systemic augmentation of alphaB-crystallin provides therapeutic benefit twelve hours post-stroke onset via immune modulation. Proceedings of the National Academy of Sciences of the United States of America. 108 (32), 13287-92 (2011).

24. Mattern, T., et al. Endotoxin and lipid A stimulate proliferation of human T cells in the presence of autologous monocytes. Journal of immunology (Baltimore, Md. 1950). 153 (7), 2996-3004 (1994).

25. Berney, T., et al. Endotoxin-mediated delayed islet graft function is associated with increased intra-islet cytokine production and islet cell apoptosis. Transplantation. 71 (1), 125-32 (2001).

26. McShane, P., Sutton, R., Gray, D.W., \& Morris, P.J. Protease activity in pancreatic islet isolation by enzymatic digestion. Diabetes. 38 Suppl 1, 126-8 (1989).

27. Ford, A.L., Goodsall, A.L., Hickey, W.F., \& Sedgwick, J.D. Normal adult ramified microglia separated from other central nervous system macrophages by flow cytometric sorting. Phenotypic differences defined and direct ex vivo antigen presentation to myelin basic proteinreactive CD4+ T cells compared. Journal of immunology (Baltimore, Md. 1950). 154 (9), 4309-21 (1995).

28. Ajami, B., Bennett, J.L., Krieger, C., Tetzlaff, W., \& Rossi, Fabio M V. Local self-renewal can sustain CNS microglia maintenance and function throughout adult life. Nature neuroscience. 10 (12), 1538-43 (2007).

29. Mildner, A., et al. Distinct and non-redundant roles of microglia and myeloid subsets in mouse models of Alzheimer's disease. The Journal of neuroscience the official journal of the Society for Neuroscience. 31 (31), 11159-71 (2011).

30. Ginhoux, F., et al. Fate mapping analysis reveals that adult microglia derive from primitive macrophages. Science (New York, N.Y.). 330 (6005), 841-5 (2010). 